\title{
Immunhistochemische Algorithmen in der Prostatadiagnostik
}

Kristiansen, G

\begin{abstract}
Zusammenfassung: Die Immunhistochemie hat sich zu einem unverzichtbaren Hilfsmittel in der Prostatabiopsiediagnostik entwickelt. Da der Basalzellverlust als ein definierendes Kennzeichen von Malignität angesehen wird, können vor allem Basalzellmarker in der Differenzialdiagnose benigner und maligner Läsionen hilfreich sein. Basalzellzytokeratine und p63 haben daher schon lange einen festen Platz im diagnostischen Repertoire von Uropathologen. Wünschenswert wäre jedoch, diese Negativmarker durch zusätzliche komplementäre Positivmarker zu ergänzen, um die diagnostische Genauigkeit weiter zu erhöhen. Der am weitesten verbreitete Positivmarker ist die -Methylazyl-CoA-Razemase (AMACR), die im Prostatakarzinom stark aufreguliert ist und sogar mit p63 in einer einzigen Immunfärbung kombiniert werden kann. Dieser Beitrag gibt einen kurzen und kritischen Überblick über gegenwärtige diagnostische Biomarker des Prostatakarzinoms und schlägt darüber hinaus Golgi-Phosphoprotein2" (GOLPH2) und "fatty acid synthase" (FASN) als zusätzliche diagnostische Marker vor
\end{abstract}

DOI: https://doi.org/10.1007/s00292-009-1230-4

Posted at the Zurich Open Repository and Archive, University of Zurich

ZORA URL: https://doi.org/10.5167/uzh-156689

Journal Article

Published Version

Originally published at:

Kristiansen, G (2009). Immunhistochemische Algorithmen in der Prostatadiagnostik. Der Pathologe, 30(S2):146-153.

DOI: https://doi.org/10.1007/s00292-009-1230-4 
Pathologe 2009 • [Suppl 2] 30:146-153

DOI 10.1007/s00292-009-1230-4

Online publiziert: 2. Oktober 2009

(c) Springer Medizin Verlag 2009

\section{G. Kristiansen}

Department Pathologie, Institut für klinische Pathologie, Universitätsspital Zürich (USZ), Schweiz

\title{
Immunhistochemische Algorithmen in der Prostatadiagnostik
}

\author{
Was gibt es Neues?
}

\begin{abstract}
Die weltweit ansteigende Inzidenz des Prostatakarzinoms, das inzwischen die häufigste maligne Neoplasie des Mannes darstellt, spiegelt sich auch in der alltäglichen Routine des diagnostischen Pathologen in der großen Zahl von Stanzbiopsien und Prostatektomiepräparaten wider [1, 2]. Die konventionelle histologische Untersuchung ist nach wie vor Goldstandard der Diagnostik; weitere molekulare prognostische oder prädiktive Marker, wie sie beispielsweise beim Mammakarzinom längst Standard sind, spielen beim Prostatakarzinom bislang keine Rolle. Dies wird sich vermutlich in der Zukunft ändern, da dringend neue Biomarker für das Karzinom benötigt werden, ein Bedarf, der durch zahlreiche Studien und neue Prognosemarkerkandidaten belegt wird [3-8].
\end{abstract}

\section{Diagnostische Anforderungen}

Um einem Teil der Prostatakarzinompatienten eine Übertherapie zu ersparen und einem anderen Teil eine intensiviertere früh einsetzende Therapie zukommen lassen zu können, ist eine individualisierte Einschätzung der Tumoraggressivität wichtig $[9,10,11]$. Bislang gibt keine verlässlichen Marker, die eine Prädiktion des Therapieansprechens je nach Therapiemodalität (Operation vs. Radiatio) ermöglichen und eine Abschätzung der entsprechenden Lebensqualität erlauben.
Dies wird in manchen prospektiven Studien untersucht, deren Daten allerdings erst in einigen Jahren vorliegen werden $[12,13]$.

Im Folgenden werden immunhistochemische Optionen in drei typischen diagnostischen Situationen erörtert. In der Biopsiediagnostik sind vor allem Marker zur Absicherung der Dignität gefordert, um auch kleinherdige Karzinominfiltrate sicher als solche zu erkennen bzw. von benignen Veränderungen abzugrenzen:

- Im Regelfall handelt es sich um nichtvorbehandelte Patienten.

- Diagnostische Schwierigkeiten können aber auch nach vorangegangener Therapie (Bestrahlung und/ oder Androgenblockade) auftreten, die das histologische Bild von Tumorund Normalgewebe verändert haben kann.

- Schließlich werden prostataspezifische Marker benötigt, um Metastasen bezüglich ihrer Herkunft abzuklären bzw. intraprostatische Metastasen und Infiltrate anderer Tumoren auszuschließen.

\section{Marker zur Sicherung der Dignität}

\section{Nichtvorbehandelte Patienten}

\section{Differenzialdiagnosen}

In Stanzbiopsien der Prostata ist ein breites Spektrum vielfältiger Differenzialdiagnosen zu beachten, die bei rein kon- ventionell-morphologischer Beurteilung Schwierigkeiten bereiten können.

Atypische Mikroacini. Sie können ein Gleason-3-Muster-Karzinom darstellen. Abzugrenzen sind vor allem dicht stehende normale Drüsen, atrophe oder partialatrophe Drüsen, eine Adenose (atypische adenomatöse Hyperplasie) oder hochgradige prostatische intraepitheliale Neoplasie- (HG-PIN-)Ausläufer. Ferner sind eine Basalzellhyperplasie, Samenblasen-I Samenleiteranteile, eine Hyperplasie des Collicus seminalis, Cowper-Drüsen oder nephrogene Reste zu erwägen.

Atypische Makroacini. Atypische Makroacini mit kribriformen oder soliden Epithelproliferaten können Anteile eines invasiven azinären „High-grade“- (Gleason-Muster-4/5-)Adenokarzinoms, eines duktalen Adenokarzinoms der Prostata oder aber eines intraduktalen Karzinoms sein. Hier gilt es, differenzialdiagnostisch eine HG-PIN, eine Basalzellhyperplasie, eine kribriforme Klarzellhyperplasie oder ein Urothelkarzinom abzugrenzen.

Infiltrative Einzelzellen. Seltener sind infiltrative Einzelzellen zu beobachten, die einem Gleason-5-Muster-Karzinom entsprechen können. Differenzialdiagnostisch ist eine Entzündung, eine hämatologische Neoplasie oder ein kleinzelliges Karzinom zu diskutieren. 
Solide Zellverbände. Bei soliden Zellverbänden kann es sich ebenso um ein Gleason-5-Muster-Karzinom handeln. Differenzialdiagnostisch kommen ein Xanthom, ein Urothelkarzinom, ein Karzinoid oder ein Paragangliom in Betracht.

Spindelzellläsionen. Spindelzellläsionen der Prostata, die insgesamt eher selten sind, können Anteile eines sarkomatoid dedifferenzierten Prostatakarzinoms sein. Differenzialdiagnostisch kommt eine myofibroblastäre Proliferation, ein Stromasarkom oder ein Leiomyosarkom infrage.

\section{Basalzellzytokeratine $34 \beta E 12$, CK5/6 und Transkriptionsfaktor p63}

Bei den oben beschriebenen Differenzialdiagnosen kann die immunhistochemische Untersuchung hilfreich sein. Seit der Erstbeschreibung von Basalzellmarkern als diagnostisches Instrument der Prostataveränderungen vor ca. 30 Jahren haben sich diese, vor allem die Basalzellzytokeratine 34ßE12 und $\mathrm{CK}_{5} / 6$ sowie der Transkriptionsfaktor p63, einen festen Platz im diagnostischen Repertoire erobert [14-19]. Invasive Prostatakarzinome sind in aller Regel, im Gegensatz zu angrenzendem benignem Parenchym, basalzellnegativ; dies macht man sich hier zu nutze. Ein typisches diagnostisches Problem mit atypischen Mikroacini in einem stark entzündlichen Hintergrund ist in - Abb. 1a gezeigt; die immunhistochemische Darstellung $\left(\mathrm{CK}_{5} / 6\right)$ lässt das basalzellnegative invasive Adenokarzinom gut erkennen (• Abb. 1b). Ob man Basalzellzytokeratine oder den Transkriptionsfaktor p63 zur Darstellung der Basalzellen bevorzugt, ist vermutlich eine Frage des Geschmacks. Nach dem Dafürhalten der Autoren ist p63 der etwas sensitivere Marker, der auch in den häufig hyperplastischen Drüsen der transitionalen Zone, die ebenfalls in Stanzbiopsien erfasst sein können, eine zuverlässigere Basalzellmarkierung gewährleistet als Basalzellzytokeratine (- Abb. 1c). Nicht allzu bekannt ist, dass Prostatakarzinome sehr selten positiv für Basalzellmarker sind (- Abb. 1d,e). Bis zu 0,3\% der Karzinome haben einer neueren Arbeit zufolge eine fokale $\mathrm{CK}_{5}$ /6-Positivität; eigene (unpublizierte) Daten zeigen in ca. $1 \%$ o der Pros-

Pathologe 2009 · [Suppl 2] 30:146-153 DOI 10.1007/s00292-009-1230-4

(c) Springer Medizin Verlag 2009

\author{
G. Kristiansen \\ Immunhistochemische Algorithmen in der Prostatadiagnostik. \\ Was gibt es Neues? \\ Zusammenfassung
}

Die Immunhistochemie hat sich zu einem unverzichtbaren Hilfsmittel in der Prostatabiopsiediagnostik entwickelt. Da der Basalzellverlust als ein definierendes Kennzeichen von Malignität angesehen wird, können vor allem Basalzellmarker in der Differenzialdiagnose benigner und maligner Läsionen hilfreich sein. Basalzellzytokeratine und p63 haben daher schon lange einen festen Platz im diagnostischen Repertoire von Uropathologen. Wünschenswert wäre jedoch, diese $\mathrm{Ne}$ gativmarker durch zusätzliche komplementäre Positivmarker zu ergänzen, um die diagnostische Genauigkeit weiter zu erhöhen. Der am weitesten verbreitete Positivmarker ist die a-Methylazyl-CoA-Razemase (AMACR), die im Prostatakarzinom stark aufreguliert ist und sogar mit p63 in einer einzigen Immunfärbung kombiniert werden kann. Dieser Beitrag gibt einen kurzen und kritischen Überblick über gegenwärtige diagnostische Biomarker des Prostatakarzinoms und schlägt darüber hinaus "Golgi-Phosphoprotein 2" (GOLPH2) und "fatty acid synthase" (FASN) als zusätzliche diagnostische Marker vor.

\section{Schlüsselwörter} Prostatakarzinom $\cdot$ Immunhistochemie . a-Methylazyl-CoA-Razemase · GolgiPhosphoprotein 2 - Fettsäuresynthase

\title{
Immunohistochemical algorithms in prostate diagnostics. What's new?
}

\section{Abstract}

Immunohistochemistry has become an indispensible tool in biopsy diagnostics of prostate tissues. In particular the use of basal cell markers can be useful to differentiate benign and malignant lesions as a lack of basal cells is considered a hallmark of malignancy. Basal cell cytokeratins and p63 have therefore a long standing place in the diagnostic portfolio of most genito-urinary pathologists. However, to complement the use of these negative markers by additional positive immunohistochemistry markers of malignancy would be desirable to further increase diagnostic accuracy. The most widely used positive marker is alpha-methylacyl-CoA racemase (AMACR), which is strongly upregulated in prostate cancer and which can even be combined with p63 in a single immunostaining. This article briefly and critically reviews current diagnostic prostate cancer biomarkers and also suggests golgi phosphoprotein 2 (GOLPH2) and fatty acid synthase (FASN) as additional diagnostic markers.

Keywords

Prostate cancer - Immunohistochemistry . a-Methylacyl-CoA racemase - Golgi phosphoprotein 2 - Fatty acid synthase 


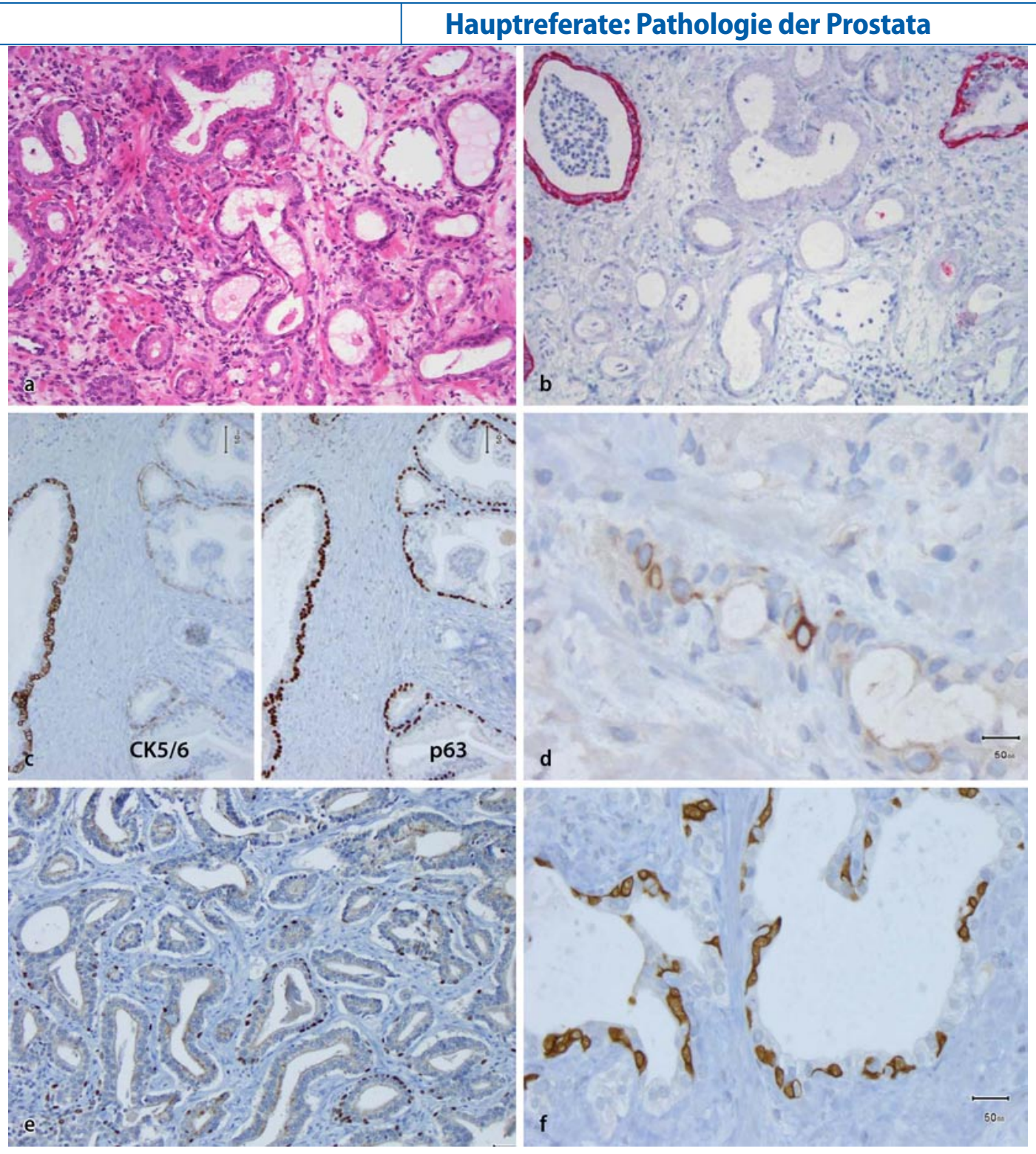

Abb. $1<$ Wert von Basalzellmarkern im Prostatakarzinom. a Entzündlich überlagertes atypisches mikroazinäres Proliferat (HE, Vergr. 200:1); b derselbe Fall, hier mit immunhistochemischer CK5/6-Darstellung (Verg. 200:1); c Vergleich von CK5/6 und p63 im Bereich des „interfaces“ zwischen peripherer Zone (jeweils links) und hyperplastischer Transitionalzone (jeweils rechts; Vergr. 200:1); d invasives Prostatakarzinom, CK5/6-Expression in einzelnen Zellen (Vergr. 400:1); e invasives Prostatakarzinom mit diskontinuierlicher p63Immunreaktivität (Vergr. 200:1); f CK5/6-p63-Doppelmarkierung (Vergr. 400:1)

tatakarzinome p63-positive Zellen [20, 21]. Häufig liegen diese Zellen in einer basalzelluntypischen, luminalen Verteilung vor. Diese Ausnahmefälle zeigen, dass man sich nicht blind auf die immunhistochemischen Befunde verlassen darf und die konventionelle morphologische Beurteilung gerade hier immer noch diagnoseweisend ist. Durch Kombination von Basalzellzytokeratinen mit p63 lässt sich eine weitere geringe Erhöhung der Sensitivität zur Detektion von Basalzellen erreichen. In - Abb. $1 f$ wird deutlich, dass einige Zellen zytoplasmatisch markiert werden, der Kern jedoch ausgespart ist; bei anderen Zellen findet sich allein das nukleäre p63-Signal. Die Mehrzahl der Zellen wird jedoch durch beide Marker markiert.

\section{a-Methylazyl-CoA-Razemase}

Neben den Basalzellmarkern hat sich in den letzten Jahren a-Methylazyl-CoA-Razemase (AMACR) als Positivmarker des Prostatakarzinoms zunehmend etabliert. Sie wurde erstmalig im Jahr 2000 als differenziell exprimiertes Transkript der Prostata beschrieben und in der Folge von zwei weiteren Arbeitsgruppen als neuer Gewebebiomarker des Prostatakarzinoms vorgeschlagen [15, 22-27]. Der immunhistochemische AMACR-Nachweis kann in der Tat sehr schöne, kontrastreiche Bilder liefern ( $\bullet$ Abb. 2a), die auch den Laien geradezu intuitiv das stark markierte invasive Karzinom erkennen lassen. Häufiger sind jedoch die Kontraste zwischen benignem und malignem Epithel nicht ganz so stark ausgeprägt (• Abb. 2b). Allerdings ist auch eine HG-PIN oder eine einfache
Hyperplasie häufig AMACR-positiv; dies schränkt den Nutzen ein (• Abb. 2c). Vor allem aber gibt es vollständig AMACRnegative Prostatakarzinome und eine erhebliche Heterogeneität in der Immunreaktivität (• Abb. 2d; [28]).

An einer großen konsekutiven Kohorte radikaler Prostatektomiepräparate ( $n=640)$ wurde eine systematische Analyse der diagnostischen Wertigkeit von AMACR durch Vergleich der Expression in Normal- und Tumorgewebe durchgeführt [29]. Hier zeigte sich in 95\% der Fälle eine stärkere Expression von AMACR im Karzinom als im angrenzenden Normalgewebe; dies belegt eindrucksvoll den diagnostischen Wert dieses Markers. Nur $5 \%$ der Fälle sind vollständig negativ für AMACR, und weitere $5 \%$ haben zumindest herdförmig vollständig AMACR- 
Abb. 2 Diagnostischer
Wert des p63-AMACRCocktails in prostatischen
Cow Geweben. a Stark AMACRpositives Prostatakarzinom (Vergr. 200:1); b mäBig stark AMACR-positives Prostatakarzinom (Vergr. 200:1); c links AMACR-Immunreaktivität in einer HG-PIN, rechts AMACRpositive hyperplastische Drüsen (Vergr. 400:1);

d AMACR-negatives, schaumzelliges Adenokarzinom der Prostata (Vergr. 400:1)

Abb. 3 > Neuer Markerkandidat im Prostatakarzinom. a TARP in Prostatageweben: links kräftige Immunreaktivität in den invasiven Karzinomdrüsen, rechts mäßig kräftige TARPExpression auch in hyperplastischen Normaldrüsen (Vergr. 200:1); b Vergleich von Fettsäuresynthase (FASN) rechts mit der korrespondierenden Stelle des p63-AMACR-Cocktails (Vergr. 200:1); c links Beispiel eines weitgehend AMACR-negativen Prostatakarzinoms, rechts korrespondierende Stelle mit der FASN-Immunreaktion (Vergr. 100:1); d Golgi-Phosphoprotein-2- (GOLPH2-) Überexpression im Prostatakarzinom (Bildmitte), angrenzend schwächere GOLPH2-Immunreaktivität in normalen Epithelien (Vergr. 200:1)
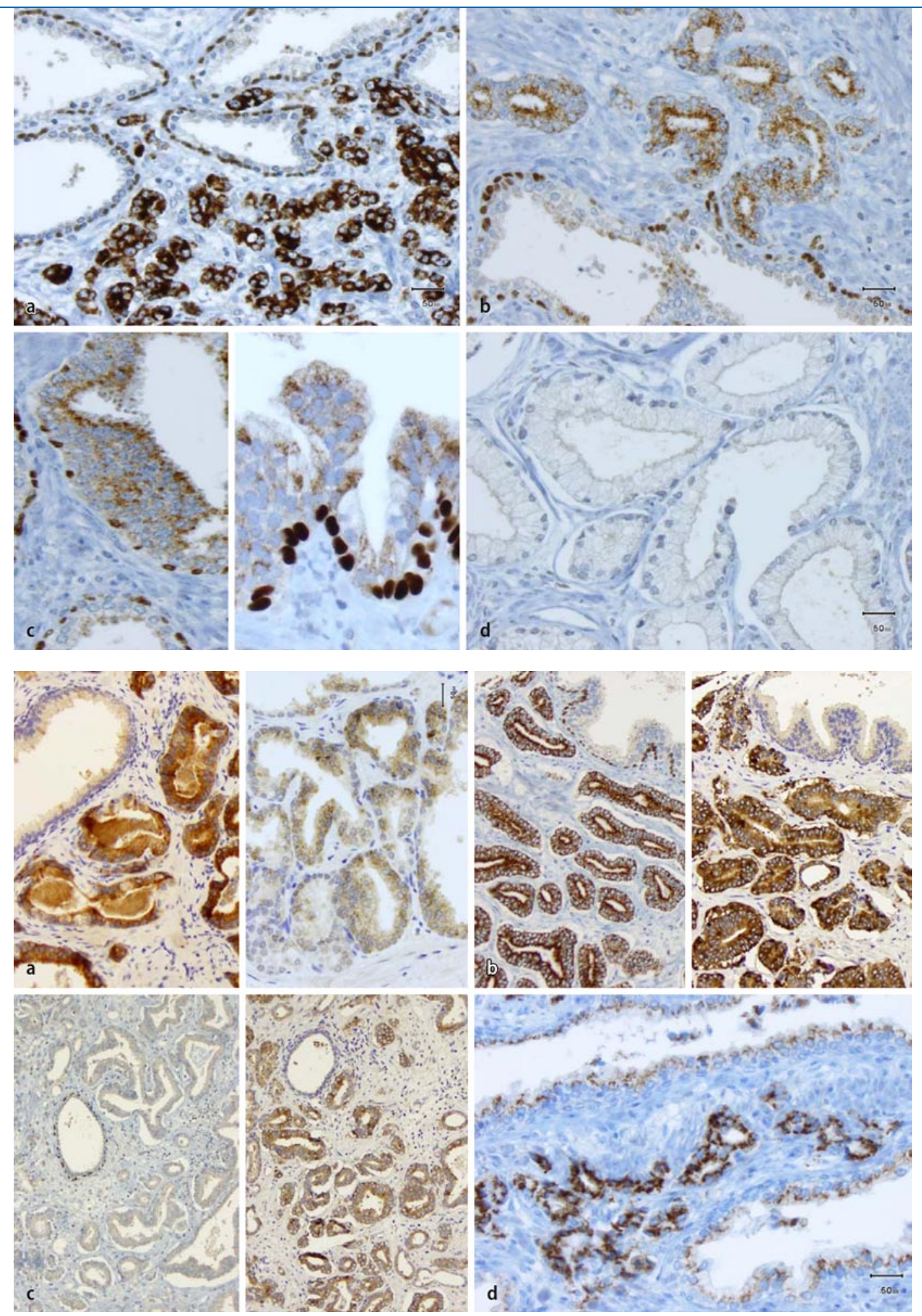

negative Anteile. Eine intratumorale Heterogenität der Expression, willkürlich definiert durch Intensitätsschwankungen um einen Bewertungspunkt in mehr als einem Viertel der untersuchten Tumorfläche, findet sich in ca. $45 \%$ der Fälle. Diese hohe Heterogenitätsrate ist natürlich pro- blematisch und wirft die Frage nach alternativen Markern auf.

\section{Weitere Markerkandidaten}

Weitere Markerkandidaten, die sich in einer vorangegangenen Studie [8] als differenziell exprimierte Transkripte des Prostatakarzinoms erwiesen hatten und da- her auch auf Proteinebene möglicherweise einen diagnostischen Nutzen haben könnten, wurden systematisch an der oben genannten Kohorte untersucht.

"TCR- $\boldsymbol{\gamma}$ alternate reading frame protein“. Das „TCR- $\gamma$ alternate reading frame protein" (TARP), ein brust- und 

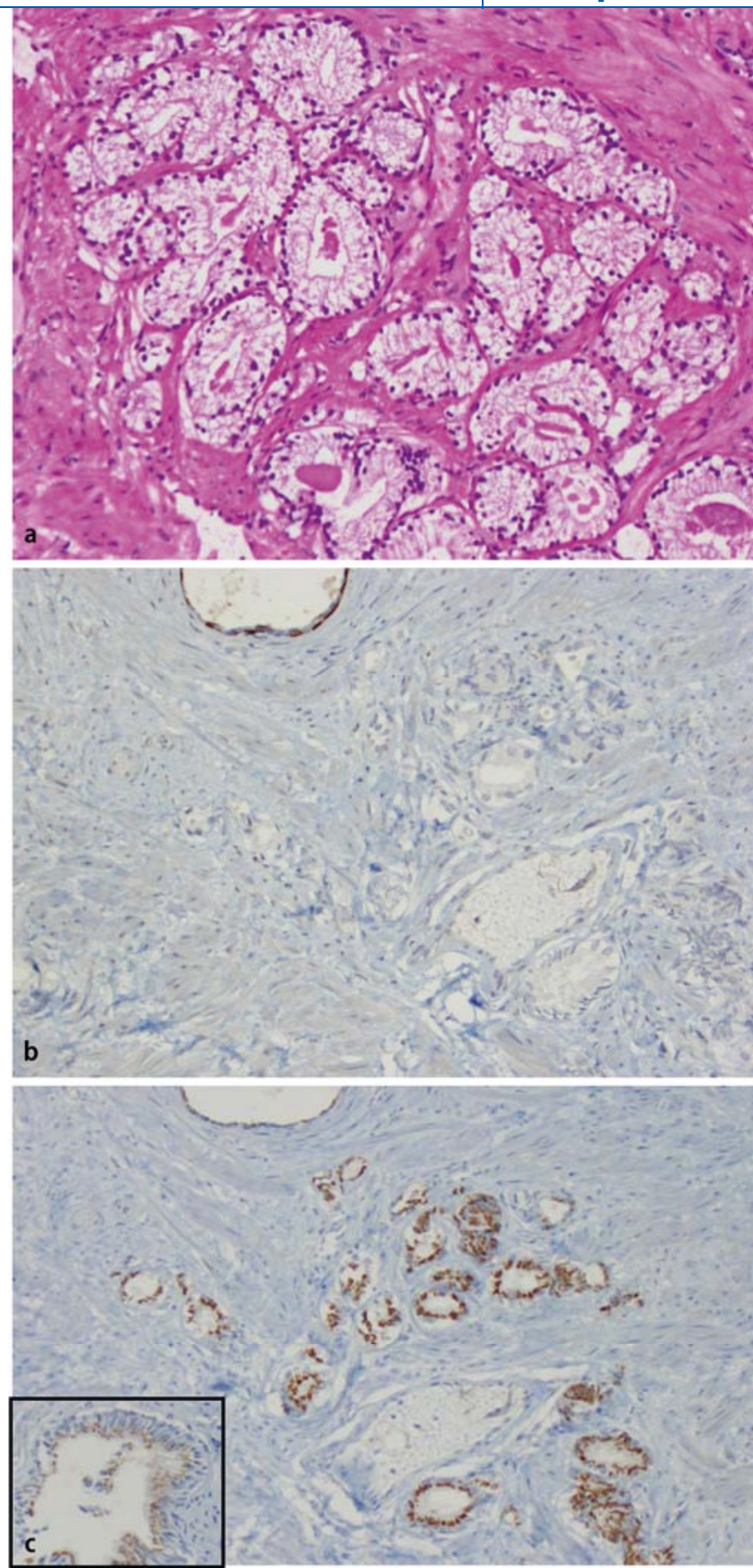

Abb. $4<$ Diagnostischer Wert von GOLPH2. a Atypisches mikroazinäres Proliferat in einem TURPSpan (HE, Vergr. 200:1), b p63-AMACR-Cocktail ohne Immunreaktivität im Vergleich zur oben im Bild dargestellten atrophen Normaldrüse; c GOLPH2-Überexpression in den atypischen Mikroacini, Einsatz unten links angrenzende normale Drüse aus der näheren Umgebung zum Vergleich (a-c Vergr. 200:1)

prostataspezifisches Antigen [30, 31], war zwar in $85 \%$ der Prostatakarzinome überexprimiert, zeigte allerdings häufig einen geringen Kontrast zwischen benignem und malignem Epithel. Seine stellenweise starke Positivität in hyperplastischen benignen Drüsen und Drüsen mit fraglicher Epitheldysplasie/"Low-grade“-PIN schränkt seinen diagnostischen Nutzen weiter ein (• Abb. 3a; Manuskript eingereicht).

Fettsäuresynthase. Die Aufregulation der Fettsäuresynthase („,fatty acid syntha-
Karzinomen durch einen FASN-Nachweis richtig identifiziert wurden.

Golgi-Phosphoprotein 2. Die Überexpression von Golgi-Phosphoprotein $2(\mathrm{GOLPH} 2)$ im Prostatakarzinom ist durch Genexpressionsanalysen schon länger bekannt; seine Eignung als Gewebebiomarker wurde jedoch erst im letzten Herbst gleich durch drei Arbeiten aufgezeigt [29, 40, 41]. Golgi-Phosphoprotein 2, dessen Funktion noch weitgehend unbekannt ist, kommt im Normalgewebe vor allem im Epithel des GolgiApparats in geringer Menge vor; im angrenzenden Prostatakarzinom lässt sich ein deutlich kräftigeres und raumgreifenderes Signal nachweisen ( $\bullet$ Abb. 3d). Ganz analog zur FASN finden sich viele Fälle, die eine gleichsinnige Aufregulation von AMACR und GOLPH2 aufweisen. Von 31 AMACR-negativen Karzinomen zeigte sich in 26 Fällen eine GOLPH2Aufregulation, die gleichfalls dessen möglichen diagnostischen Nutzen begründet. Schwächen von GOLPH2 sind die starke Überexpression auch in hyperplastischem Normalgewebe und seine Expression in jeglichem Normalgewebe, sodass allein eine vergleichende Untersuchung von zweifelsfrei als benigne $\mathrm{zu}$ identifizierenden Drüsen mit fraglich karzinomsuspekten Drüsen eine diagnostisch wertvolle Hilfe sein kann. Im Vergleich zu Basalzellmarkern oder AMACR stellt dies somit andere Anforderungen an die morphologische Expertise des Untersuchers. In • Abb. 3e ist ein Beispiel eines AMACR-negativen, jedoch stark GOLPH2-positiven Karzinoms der Prostata dargestellt.

\section{Schlussfolgerungen}

Die eigenen präliminären Analysen zeigen, dass GOLPH2 und FASN sich als neue diagnostische Positivmarker des Prostatakarzinoms eignen. Beide detektieren einzeln jeweils über $92 \%$ aller Karzinomfälle. In Kombination mit p63 und AMACR werden über $99 \%$ der malignen Drüsen als solche richtig zugeordnet. Eine weitere Validierung dieser Marker an seltenen Karzinomsubtypen und insbesondere an benignen Krebsimitatorläsionen erfolgt im Rahmen der täglichen Routineanwendung im Universitätsspital in Zürich. 


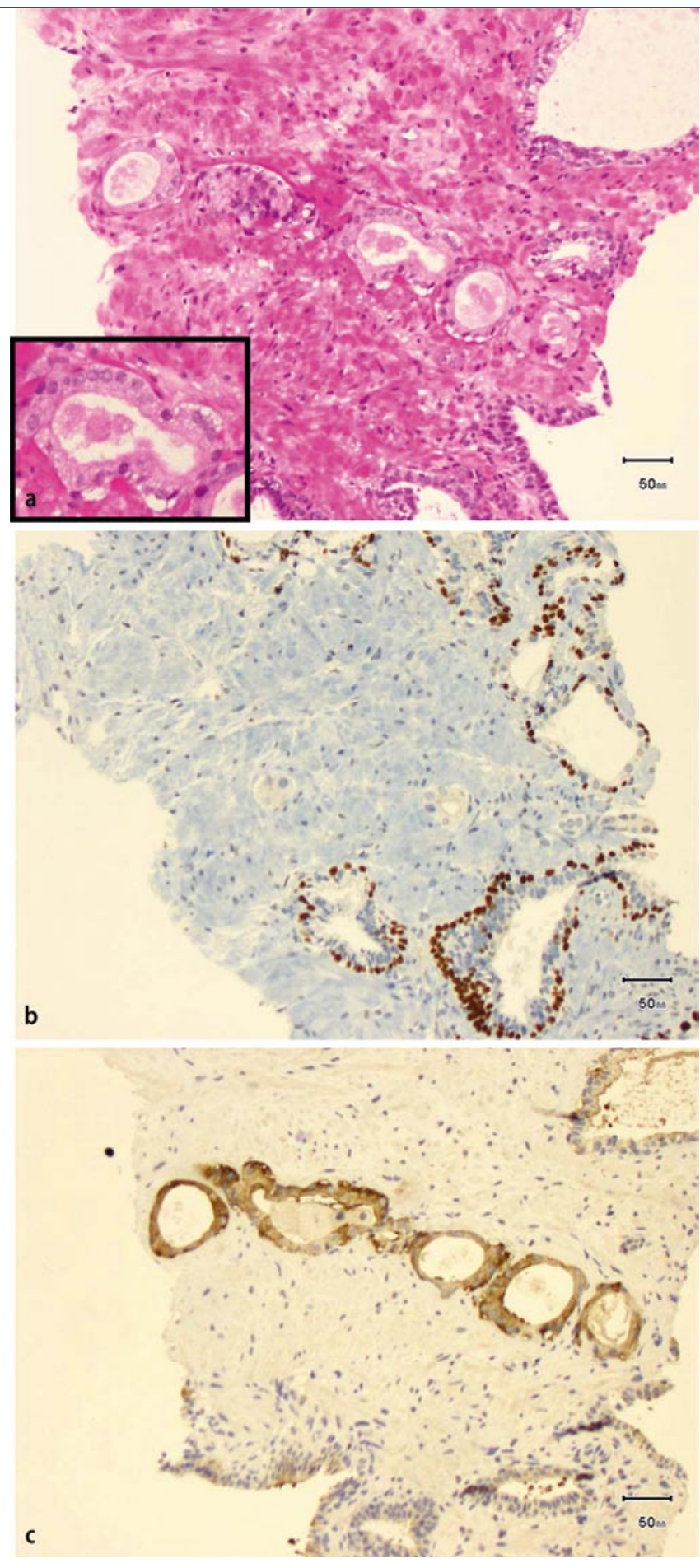

Abb. $5 \Delta$ Diagnostischer Wert der FASN. a Atypisches mikroazinäres Proliferat mit Kernatypie (Einsatz unten links); b korrespondierende Stelle, immunhistochemischer Befund mit p63-AMACR-Cocktail: keine Nachweise einer Basalzelllage, jedoch auch keine AMACR-Immunreaktivität; $c$ weiterer konsekutiver Schnitt, FASN-Immunreaktion: deutliche Markierung der atypischen Drüsen, hingegen nur geringe Reaktion in den angrenzenden normalen Drüsen (a-c Vergr. 200:1)

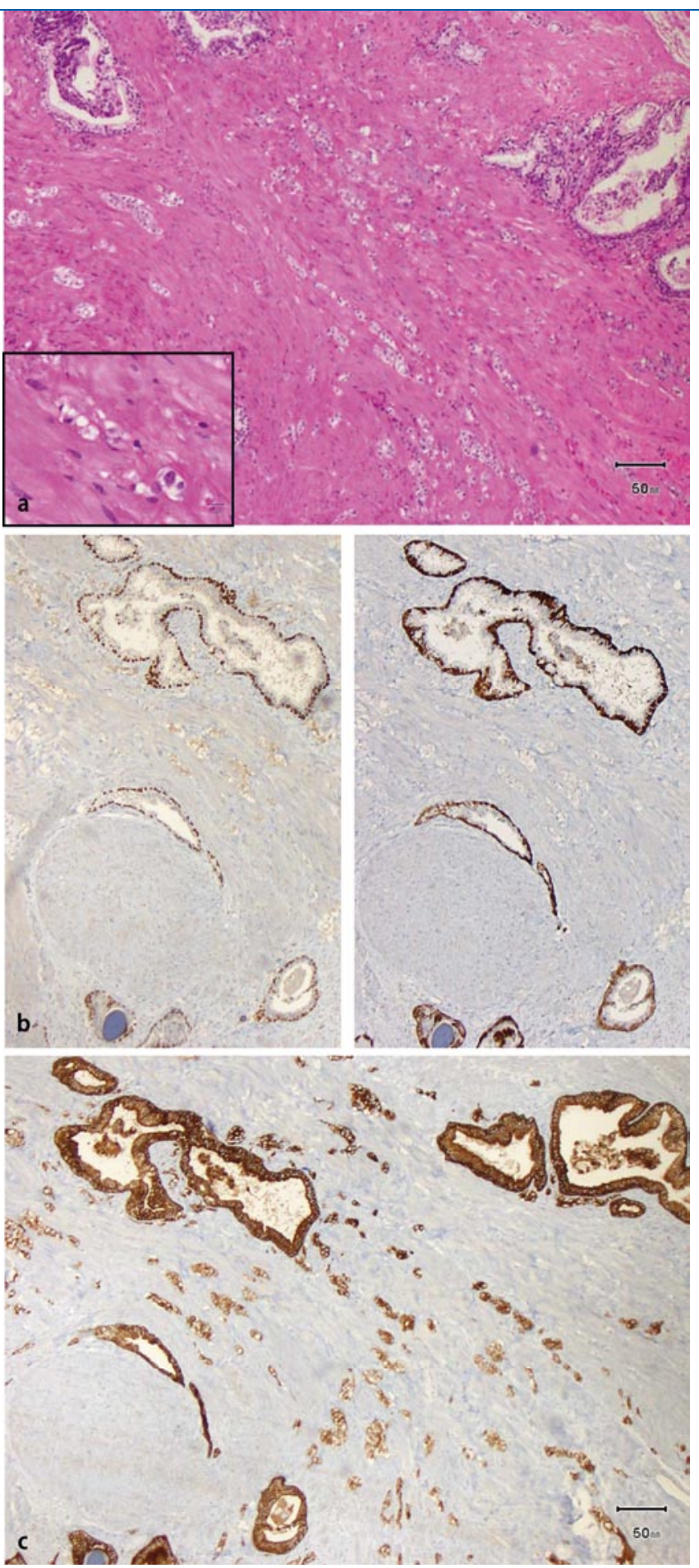

Abb. $6 \Delta$ Antiandrogen behandeltes Adenokarzinom der Prostata (HEFärbung). a Einsatz unten links deutlich sichtbare zytolytische Therapieeffekte; $\boldsymbol{b}$ links p63-AMACR-Nachweis mit vollständigem Basalzellverlust und wesentlicher AMACR-Überexpression, rechts fast noch schönerer Kontrast im CK5/6-Nachweis; c Nachweis des Basalzellzytokeratins (AE1/AE3), das die invasiven, therapiebedingt alterierten Drüsen deutlich hervortreten lässt (a-c Vergr. 100:1)

\section{Beispiele aus der Praxis}

In $-\mathbf{A b b} .4 \mathrm{a}$ ist ein kleines atypisches mikroazinäres Proliferat, das sich unifokal in TURP-Materialien fand, dargestellt. In den weiteren Schnittstufen für die immunhistochemische Untersuchung war dieses Areal nur noch teilweise erfasst worden, daher ergibt sich die inkongruente Dar- stellung der immunhistochemischen p63/AMACR-Nachweise, die zwar einen deutlichen Verlust der p63-Immunreaktivität, nicht jedoch eine Aufregulation 


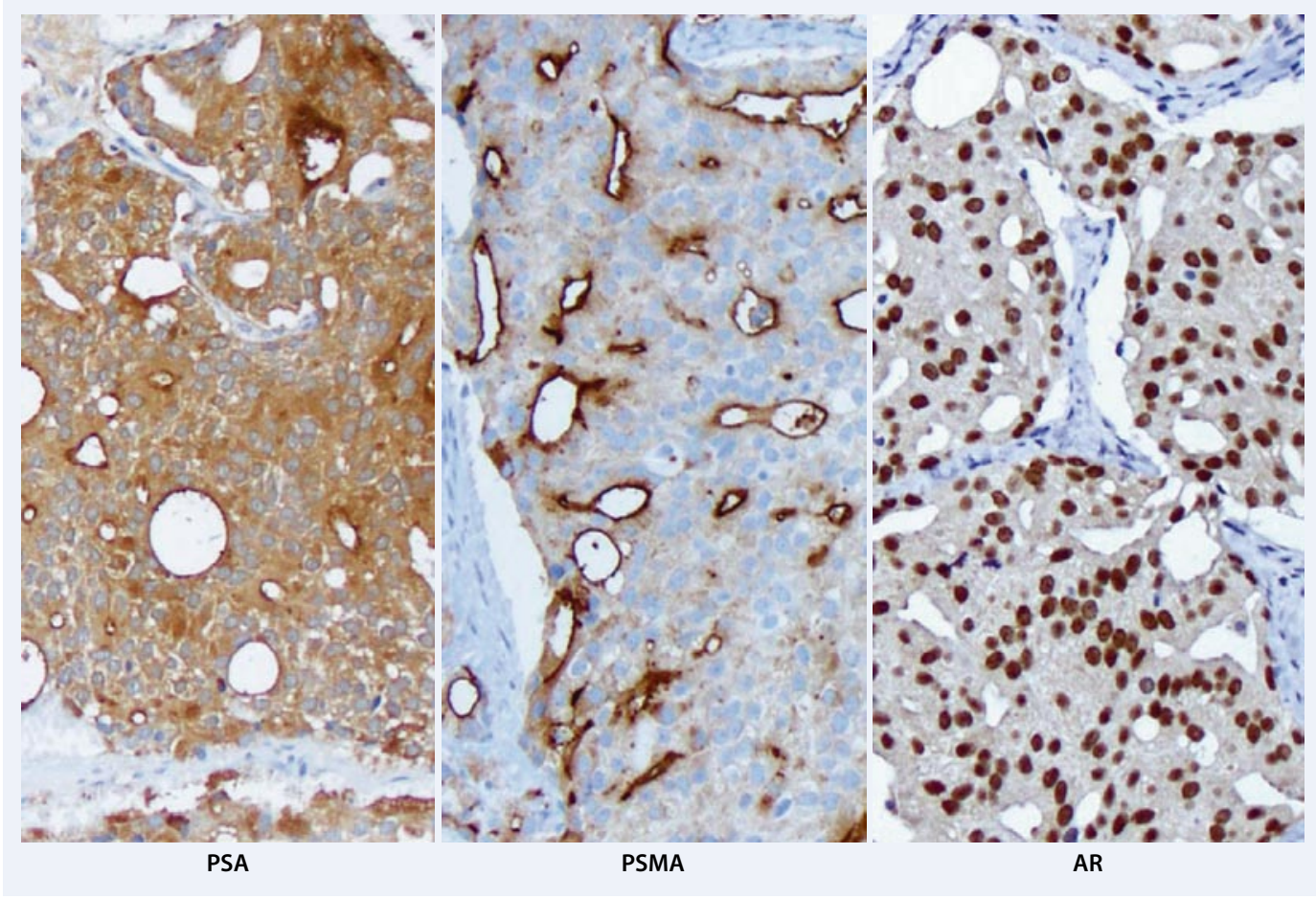

Abb. $7<$ Zervikale Metastase eines Adenokarzinoms der Prostata. Links starke Überexpression des prostataspezifischen Antigens (PSA); Bildmitte kräftige und membranständige Expression des prostataspezifischen Membranantigens (PSMA); rechts zusätzlich kräftige Androgenrezeptor- (AR-)Expression (alle Vergr. 200:1)

von AMACR zeigt. Im immunhistochemischen GOLPH2-Nachweis eines konsekutiven Schnittes (- Abb. 4c) zeigt sich jedoch deutlich die kräftige Überexpression von GOLPH2 im Vergleich zu angrenzenden Normaldrüsen (• Abb. 4, Einsatz unten links).

Im nächsten Beispiel eines Stanzbiopsats (• Abb. 5a, unten links) findet sich ein kleiner Ausläufer atypischer Mikroacini mit eindeutiger Kernatypie. Der immunhistochemische AMACR-Befund zeigt bei unvollständiger Darstellung der noch erhaltenen Drüsen keine Basalzellen, jedoch auch keine AMACR-Überexpression. Eindeutig fällt dagegen das Ergebnis der FASN-Immunreaktion aus (- Abb. 5c), sodass sich auch hier ein Adenokarzinom der Prostata sichern lässt.

\section{Zürcher Schema zur Diagnostik des Prostatakarzinoms}

Das Zürcher Schema zur Diagnose des Prostatakarzinoms basiert zunächst auf einer konventionellen Analyse der morphologischen Strukturen, gefolgt von einer kombinierten p63-AMACR-Immunreaktion (Kombination von AMACR-Kaninchen polyklonal; Fa. Biologo, Kronshagen, Deutschland, Verdünnung 1:200. p63-Klon-Mischung 4A4-Y4A3; Fa. Neomarkers Fremont, USA), gefolgt von einer
FASN- (Klon ${ }_{3} \mathrm{~F}_{2}-1 \mathrm{~F}_{3}$, Fa. Abnova Corp., Taipei, Taiwan) und GOLPH2-Immunreaktion (Klon 5B10; Fa. Abnova Corp., Taipei, Taiwan). Mit dieser Kombination gelingt es in den allermeisten Fällen, zu einer diagnostischen Aussage zu kommen. Einschränkend muss jedoch angemerkt werden, dass insbesondere FASN und GOLPH2 beide noch nicht hinreichend an Karzinomimitatorläsionen validiert sind. Streng genommen, trifft dies auch für den inzwischen weit verbreiteten Marker AMACR zu, dem neuere Arbeiten eine positive Immunreaktion auch in der partialen Atrophie der Prostata, einer benignen Läsion, in bis zu 30\% der Fälle bescheinigen [42].

\section{Vorbehandelte Prostatakarzinome}

Die seltenere Situation eines behandelten Prostatakarzinoms, sei es nach androgener Blockadetherapie oder nach stattgehabter Radiatio, ist meist diagnostisch einfacher, auch wenn Therapieeffekte erhebliche Regressionszeichen des Karzinoms hervorrufen können (• Abb. 6a). Hier findet sich ebenfalls ein Verlust von Basalzellmarkern, allerdings auch häufig von AMACR (• Abb. 6b). Die invasive Natur der Läsion lässt sich jedoch in den meisten Fällen durch ein Panzytokeratin (• Abb. 6c) zweifelsfrei belegen.
Diesem Antikörper kommt daher die wesentlichere Bedeutung nach den Basalzellmarkern zu.

\section{Beurteilung von Metastasen}

Die dritte diagnostische Situation, in der prostataspezifische Marker zum Einsatz kommen, liegt in der Abklärung von Metastasen bzw. der Absicherung des Primarius. Hier hat sich das prostataspezifische Antigen (PSA) als tatsächlich weitgehend prostataspezifisch gut bewährt. Allerdings ist es gerade bei zunehmender Tumordedifferenzierung häufig nur schwach exprimiert oder kann gar ganz verloren gehen, was seine relativ geringe Sensitivität begründet. Hier ist in den letzten Jahren das prostataspezifische Membranantigen (PSMA) vielfach als weiterer Marker empfohlen und verwendet worden. Dies ist insofern sinnvoll, als das PSMA gerade mit zunehmender Tumordedifferenzierung aufreguliert und auch in Metastasen zumeist kräftig exprimiert wird. Nachteilig ist jedoch die fehlende Prostataspezifität, da auch andere Tumoren, wie z. B. papilläre Nierenzellkarzinome, Blasenkarzinome und gastrointestinale Karzinome PSMA-positiv sein können [43]. Am Universitätsspital Zürich (USZ) wird die Kombination aus PSA, PSMA und dem Androgenrezeptor, der in den allermeis- 
ten Prostatakarzinomen überexprimiert wird, verwendet (• Abb. 7).

\section{Fazit für die Praxis}

Immunhistochemische Untersuchungen spielen in der Prostatadiagnostik eine zunehmend wichtigere Rolle; sie dürfen jedoch nicht unkritisch und nicht ungeachtet des morphologischen Kontextes eingesetzt werden. Neben Basalzellmarkern (Zytokeratinen und p63) sowie AMACR können auch FASN oder GOLPH2 diagnostisch nützlich sein. Das Zürcher Schema zur Diagnostik des Prostatakarzinoms basiert zunächst auf einer konventionellen Analyse der morphologischen Strukturen, gefolgt von einer Kombination aus p63-AMACR-, FASNund GOLPH2-Immunreaktion. Hiermit gelingt es in den allermeisten Fällen, zu einer diagnostischen Aussage zu kommen. Je nach diagnostischer Fragestellung und morphologischem Kontext kann auch mit weiteren Markern kombiniert werden, um Metastasen abzuklären sowie andere Primärtumoren auszuschließen oder zu bestätigen. Die Beschreibung weiterer prostataspezifischer Marker wäre wünschenswert.

\section{Korrespondenzadresse \\ Prof. Dr. G. Kristiansen}

Department Pathologie, Institut für klinische Pathologie, Universitätsspital Zürich (USZ) Schmelzbergstr. 12, 8091 Zürich

Schweiz

glen.kristiansen@usz.ch

Interessenkonflikt. Der korrespondierende Autor gibt an, dass kein Interessenkonflikt besteht.

\section{Literatur (Auswahl)}

2. Jemal A, Siegel R, Ward E et al (2009) Cancer statistics, 2009. CA Cancer J Clin 59:225-249

3. Dhanasekaran SM, Barrette TR, Ghosh D et al (2001) Delineation of prognostic biomarkers in prostate cancer. Nature 412:822-826

8. Kristiansen G, Pilarsky C, Wissmann C et al (2005) Expression profiling of microdissected matched prostate cancer samples reveals CD166/MEMD and CD24 as new prognostic markers for patient survival. J Pathol 205:359-376

10. Klotz L (2007) Active surveillance for favorable-risk prostate cancer: who, how and why? Nat Clin Pract Oncol 4:692-698

12. Umbehr M, Kessler TM, Sulser T et al (2008) ProCOC: the prostate cancer outcomes cohort study. BMC Urol 8:9
14. Brawer MK, Peehl DM, Stamey TA, Bostwick DG (1985) Keratin immunoreactivity in the benign and neoplastic human prostate. Cancer Res 45:36633667

15. Epstein Jl (2004) Diagnosis and reporting of limited adenocarcinoma of the prostate on needle biopsy. Mod Pathol 17:307-315

18. Signoretti S, Waltregny D, Dilks J et al (2000) p63 is a prostate basal cell marker and is required for prostate development. Am J Pathol 157:1769_ 1775

20. Osunkoya AO, Hansel DE, Sun X et al (2008) Aberrant diffuse expression of p63 in adenocarcinoma of the prostate on needle biopsy and radical prostatectomy: report of 21 cases. Am J Surg Pathol 32:461-467

21. Ali TZ, Epstein Jl (2008) False positive labeling of prostate cancer with high molecular weight cytokeratin: p63 a more specific immunomarker for basal cells. Am J Surg Pathol 32:1890-1895

22. Xu J, Stolk JA, Zhang $X$ et al (2000) Identification of differentially expressed genes in human prostate cancer using subtraction and microarray. Cancer Res 60:1677-1682

24. Rubin MA, Zhou M, Dhanasekaran SM et al (2002) alpha-Methylacyl coenzyme A racemase as a tissue biomarker for prostate cancer. JAMA 287:1662-1670

28. Murphy AJ, Hughes CA, Lannigan G et al (2007) Heterogeneous expression of alpha-methylacyl$\mathrm{CoA}$ racemase in prostatic cancer correlates with Gleason score. Histopathology 50:243-251

29. Kristiansen G, Fritzsche FR, Wassermann K et al (2008) GOLPH2 protein expression as a novel tissue biomarker for prostate cancer: implications for tissue-based diagnostics. Br J Cancer 99:939-948

30. Maeda H, Nagata S, Wolfgang CD et al (2004) The $T$ cell receptor gamma chain alternate reading frame protein (TARP), a prostate-specific protein localized in mitochondria. J Biol Chem 279:2456124568

31. Wolfgang CD, Essand M, Vincent JJ et al (2000) TARP: a nuclear protein expressed in prostate and breast cancer cells derived from an alternate reading frame of the T cell receptor gamma chain locus. Proc Natl Acad Sci U S A 97:9437-9442

32. Epstein JI, Carmichael M, Partin AW (1995) OA-519 (fatty acid synthase) as an independent predictor of pathologic state in adenocarcinoma of the prostate. Urology 45:81-86

33. Shurbaji MS, Kalbfleisch JH, Thurmond TS (1996) Immunohistochemical detection of a fatty acid synthase (OA-519) as a predictor of progression of prostate cancer. Hum Pathol 27:917-921

34. Swinnen JV, Roskams T, Joniau S et al (2002) Overexpression of fatty acid synthase is an early and common event in the development of prostate cancer. Int J Cancer 98:19-22

35. Rossi S, Graner E, Febbo P et al (2003) Fatty acid synthase expression defines distinct molecular signatures in prostate cancer. Mol Cancer Res 1:707715

36. Baron A, Migita T, Tang D, Loda M (2004) Fatty acid synthase: a metabolic oncogene in prostate cancer? J Cell Biochem 91:47-53

37. Shah US, Dhir R, Gollin SM et al (2006) Fatty acid synthase gene overexpression and copy number gain in prostate adenocarcinoma. Hum Pathol 37:401-409

38. Di Vizio D, Sotgia F, Williams TM et al (2007) Caveolin-1 is required for the upregulation of fatty acid synthase (FASN), a tumor promoter, during prostate cancer progression. Cancer Biol Ther 6:12631268
39. Migita T, Ruiz S, Fornari A et al (2009) Fatty acid synthase: a metabolic enzyme and candidate oncogene in prostate cancer. J Natl Cancer Inst 101:519-532

40. Wei S, Dunn TA, Isaacs WB et al (2008) GOLPH2 and MYO6: putative prostate cancer markers localized to the Golgi apparatus. Prostate 68:1387-1395

41. Varambally S, Laxman B, Mehra R et al (2008) Golgi protein GOLM1 is a tissue and urine biomarker of prostate cancer. Neoplasia 10:1285-1294

42. Wang W, Sun X, Epstein JI (2008) Partial atrophy on prostate needle biopsy cores: a morphologic and immunohistochemical study. Am J Surg Pathol 32:851-857

43. Kinoshita Y, Kuratsukuri K, Landas S et al (2006) Expression of prostate-specific membrane antigen in normal and malignant human tissues. World J Surg 30:628-636

Das vollständige Literaturverzeichnis ...

... finden Sie in der html-Version dieses Beitrags im Online-Archiv auf der Zeitschriftenhomepage www.DerPathologe.de 